\title{
Molecular Characterization of Fermenting Yeast Species from Fermented Teff Dough during Preparation of Injera Using ITS DNA Sequence
}

\author{
Belay Tilahun Tadesse $\mathbb{D}^{\mathrm{D}},{ }^{1}$ Andualem Bahiru Abera, ${ }^{2}$ Anteneh Tesfaye Tefera ${ }^{\mathbb{D}}{ }^{3}$ \\ Diriba Muleta $\mathbb{D}^{\mathrm{D}}{ }^{3}$ Zewdu Terefework Alemu, $^{4}$ and Gary Wessel ${ }^{5}$ \\ ${ }^{1}$ Wolkite University, Collage of Natural and Computational Science, Biotechnology Department, Ethiopia \\ ${ }^{2}$ Addis Ababa Institute of Technology, Addis Ababa University, Ethiopia \\ ${ }^{3}$ Institute of Biotechnology, Addis Ababa University, Ethiopia \\ ${ }^{4}$ MRC-ET Molecular Diagnostics, Addis Ababa, Ethiopia \\ ${ }^{5}$ Department of Molecular Biology, Cellular Biology and Biochemistry, Brown University, Providence, RI 02912, USA
}

Correspondence should be addressed to Belay Tilahun Tadesse; belayt21@gmail.com

Received 15 February 2019; Accepted 7 April 2019; Published 1 July 2019

Academic Editor: Alejandro Castillo

Copyright (C) 2019 Belay Tilahun Tadesse et al. This is an open access article distributed under the Creative Commons Attribution License, which permits unrestricted use, distribution, and reproduction in any medium, provided the original work is properly cited.

\begin{abstract}
Identification of the yeast responsible for Injera fermentation is important in order to be more consistent and for scale-up of Injera production. In this study, yeast were isolated and identified from fermenting teff dough sample collected from household, hotels, and microenterprises, Addis Ababa. Initially, the yeast obtained from fermenting teff dough of different sources were selected on the basis of their $\mathrm{CO}_{2}$ production potentials. Its DNA sequencing of isolated yeast identified Pichia fermentans, Pichia occidentalis, Candida humilis, Saccharomyces cerevisiae, and Kazachstania bulderi. The association of identified yeast to their sources indicated the presence of Pichia fermentans in fermenting dough samples collected from all sources whereas Kazachstania bulderi, Saccharomyces cerevisiae, and Candida humilis were shown to be present in samples collected from households, hotels, and microenterprises, respectively. The phenotypes and $\mathrm{CO}_{2}$ production potentials of this yeast were also documented. This study has confirmed the presence of different yeast species in the fermentation of teff dough and hinted the complex nature of Injera dough fermentation.
\end{abstract}

\section{Introduction}

Fermentation, degradation of organic compounds without net oxidation by the microbial, is an important process in production and preservation of food. It is one of the oldest food-processing technologies known, with some records dating back to 6,000 B.C. [1]. Locally with available raw materials from plant or animal sources, people around the world prepare fermented food and drink either naturally or by the addition of microbial starter cultures using traditional knowledge. Micro-organisms, by virtue of their metabolic activities, transform these raw materials both biochemically (i.e., the nutrients) and organoleptically (i.e., the taste/texture/odor, visual appearance) into appealing products that are culturally acceptable to the maker and consumer, which at the same time have improved shelf-life and safety $[2,3]$. In addition, the unique group of microbiota increases the levels of protein, vitamin, essential amino acids, fatty acids, digestibility, and pharmacological values. The importance of microbiota in modern-day life is underlined by the wide spectrum of fermented foods marketed in industrialized and developing countries [2]. This also demonstrates that these products play potential role in securing the food demands of people in developing countries [4].

In developing countries food security is a major challenge for many reasons. A traditional way of food production practice is among the major cause because it results in poor yield, inefficient raw material processing, and loss due 
to poor management, spoilage, and contamination. This mainly holds true regarding food production practices of traditionally fermented foods. In general, in developing countries, most of the traditional fermented foods are made under primitive conditions of spontaneous (uncontrolled) fermentations of nonsterile state which results in low yield and poor quality food products [5]. The conditions, besides having a positive contribution to be important sources of the natural fermenting microorganisms, unfortunately, are exposing the fermented food products to contaminating and spoiling microorganisms that can survive the fermentation conditions [3] and the major sources of diarrheal disease [6] and food spoilage $[7,8]$, exacerbating poor socioeconomic conditions and taxing the nutritional requirements of the society.

Yeast are the most important microorganism in fermented foods and beverages such as bread, Injera, alcoholic beverages, cheeses, and production of biologically important products like insulin, enzyme, vitamins, and antibiotics [9, 10]. Yeast are unicellular, eukaryotic, and polyphyletic organisms classified in the kingdom fungi. They are ubiquitous and commonly found on fruits, bulla, kocho, vegetables, and other plant materials [11]. However, yeast are highly involved in food fermentations, but information on the dominant yeast species in African fermented foods still needs extra engagement especially in Ethiopian fermented teff dough $[12,13]$. Thus, the identity of the proper yeast communities for each fermented product is essential.

In Ethiopia most of the research conducted on traditional fermented food primarily focuses on the determination of the microorganisms which are involved in the fermentation, spoilage, and contamination of the food. Unfortunately, limited research has been done on Injera, a fermented teff product of major importance in the nutrition and tradition of Ethiopians. Current research reports are largely limited to only investigating microbial ecology of Injera employing only phenotypic characterization [12, 14-17]. A very limited literature is available on identification of yeast in teff dough fermentation using molecular approach $[18,19]$. This research work aims to identify yeast using molecular approaches to gain a rapid and accurate identification of yeast using intertranscribed spacer (ITS) DNA of yeast [20]. We anticipate this better understanding of yeast microbial communities found in fermented teff dough will permit crafting of unique characteristics of Injera to enhance its consumption and efficiency in production world-wide.

\section{Materials and Methods}

2.1. Sample Collection and Description of the Study Sites. Teff dough samples (two hundred gram each, 97 total) were collected from hotels, households and Injera baking microenterprises in Addis Ababa, Ethiopia. Sample processing, laboratory isolation, and identification of yeast were carried out in the Holeta Biotechnology Institute, Microbial Biotechnology Laboratory. Molecular characterization was carried out at the MRC-ET Molecular Diagnostics Laboratory, Addis Ababa, Ethiopia and DNA sequencing was performed at Genewiz (South Plainfield, New Jersey, USA).
2.2. Isolation and Selection of Yeast. Samples of dough were serial diluted by taking $10 \mathrm{~g}$ of dough from each sample into separate flasks with $90 \mathrm{~mL}$ sterile $0.1 \%$ peptone water and then homogenized. From this preparation, $1 \mathrm{~mL}$ was diluted in to $9 \mathrm{ml}$ sterile distilled water; then $0.1 \mathrm{~mL}$ of appropriate dilution of separate samples was spread onto presolidified Yeast Extract Glucose Chloramphenicol Bromophenol Blue Agar plates (i.e., yeast extract $(5.0 \mathrm{~g} / \mathrm{L})$, glucose $(20.0 \mathrm{~g} / \mathrm{L})$ Chloramphenicol $(0.1 \mathrm{~g} / \mathrm{L})$, Bromophenol blue $(0.01 \mathrm{~g} / \mathrm{L}))$ and incubated at $37^{\circ} \mathrm{C}$ for $24 \mathrm{~h}$. After incubation, representative yeast colonies were selected and subcultured using the same media three times to purify the isolates. Based on their cultural characteristics (colony size, colony color, and colony texture) 155 yeast isolates were selected from each starting culture. Gas $\left(\mathrm{CO}_{2}\right)$ production from glucose was used as a preliminary selection test criterion to further screen the isolates [21].

2.3. Production of Gas $\left(\mathrm{CO}_{2}\right)$ from Glucose. A total of 155 yeast isolates were inoculated into tubes containing yeast extract $(5.0 \mathrm{~g} / \mathrm{L})$ glucose $(20.0 \mathrm{~g} / \mathrm{L})$ broth and were incubated at $37^{\circ} \mathrm{C}$ for $24 \mathrm{~h}$. Production of gas $\left(\mathrm{CO}_{2}\right)$ from glucose was assayed using $1.5 \%$ peptone water to which $1 \%$ glucose was $20.0 \mathrm{~g} / \mathrm{L}$ and $1 \mathrm{~mL}$ of fresh Andrade indicator solution per $100 \mathrm{~mL}$ distilled water was added. The medium was distributed in test tubes containing inverted Durham tubes. The medium was autoclaved at $121^{\circ} \mathrm{C}$ for 15 minutes and was then inoculated with the required cultures. Sterile paraffin wax of $2 \mathrm{ml}$ was overlaid in each test tube to prevent the entry of oxygen. All tubes were incubated at $37^{\circ} \mathrm{C}$ and were examined daily for gas production for 7 days [22].

\subsection{Molecular Characterization of Microbes}

2.4.1. Genomic DNA Extraction. The genomic DNA of isolates was extracted using an Invisorb ${ }^{\circledR}$ Spin Genomic DNA Extraction kit according to the instruction of the manufacturer $[22,23]$.

2.4.2. Amplification of ITS DNA of Yeast Isolates. Regions of each yeast internal transcribed spacers (ITS1 and ITS2) of DNA extract was amplified using nested primers ITS1 and ITS2 [16]. For ITS DNA region, PCR amplification reaction mixtures $(50 \mu \mathrm{L})$ contained $1 \mu \mathrm{L}$ of the extracted DNA, $1 \mu \mathrm{L}$ dNTPs, $1 \mu \mathrm{L}$ of each of primers ITS1 (-TCC GTA GGT GAA CCT GCG G-) and ITS2 (-GCT GCG TTC TTC ATC GAT GC-), $1 \mu \mathrm{L}$ of Taq DNA polymerase (1.25 units/50 $\mu \mathrm{L}$ ) (Fermentas, St. Leon- Rot, Germany), and $5 \mu \mathrm{L}$ PCR buffer. The final reaction volume was adjusted to $50 \mu \mathrm{l}$ by adding reverse osmosis purified water. The PCR cycle conditions include an initial denaturation step at $95^{\circ} \mathrm{C}$ for $10 \mathrm{~min}$, followed by 35 cycles of denaturation at $94^{\circ} \mathrm{C}$ for $30 \mathrm{Sec}$, primer annealing at $55^{\circ} \mathrm{C}$ for $1 \mathrm{~min}$, and primer extension at $72^{\circ} \mathrm{C}$ for $1 \mathrm{~min}$ with a final extension at $72^{\circ} \mathrm{C}$ for $5 \mathrm{~min}$ [16]. PCR product then was separated by gel electrophoresis using a 3\% Agarose gel and $1 \mu \mathrm{L}$ loading dye with $5 \mu \mathrm{L}$ PCR products and stained with Ethidium bromide for gel documentation. 
2.4.3. Cloning and Sequencing of ITS DNA of Yeast. The PCR amplified a genomic region of interest (i.e., ITS DNA of yeast) of each isolate was cloned into pGEMT EZ vector, transformed into XL1-blue cells, and inoculated onto plated LB medium containing $100 \mu \mathrm{g} / \mathrm{mL}$ ampicillin for selection according to the manufacturer's instructions (Promega Corporation). Isolates were grown for $16 \mathrm{~h}$ at $37^{\circ} \mathrm{C}$ with vigorous shaking in $\mathrm{LB} / \mathrm{Amp}$ and the resulting plasmids were isolated as described in the QIA quick Gel Extraction Kit. DNA was then sequenced by automated DNA sequencer (ABI model 377; Applied Biosystems) at Genewiz (South Plainfield, NJ USA).

2.4.4. Phylogenetic Analysis. DNA sequences were edited, and consensus sequences were obtained using the Bioedit software package http://www.mbio.ncsu.edu/BioEdit/bioedit .html. Final sequences were then aligned using CLUSTAL (version: 1.2.4) [24] for each of the sequences. The sequences of yeast isolates of this study were then compared to those in GenBank (National Centre for Biotechnology Information; https://blast.ncbi.nlm.nih.gov/Blast.cgi) using the Basic Local Alignment Search Tool [25] for nucleotide sequences (blastn). Evolutionary analyses were conducted using the Maximum Likelihood method based on the Tamura-Nei model by MEGA7 [26, 27].

2.4.5. Isolates Designation. Microbial isolates were designated as follows: AAUYT for yeast isolate followed by numbers and capital letters $A, B$, and $C$ which represent the range of time of fermentation. A represents $48 \mathrm{~h}, \mathrm{~B}$ represents $60 \mathrm{~h}$, and $\mathrm{C}$ represents $72 \mathrm{~h}$ of fermentation.

2.4.6. Data Analysis. All data were analyzed by using the statistical data analysis software SPSS 20.0 version. And the phylogenetic tree construction was accomplished by MEGA7 software.

\section{Results}

3.1. Isolation of Yeast and Gas Production from Glucose. The yeast isolates $(n=155)$ recovered were observed to have different features of colonial morphology. The yeast isolates showed diverse culture characteristics with regard to color (white, gray pigmentation, or blue), shape (circular or irregular), edge (irregular or smooth), and elevation (flat or raised).

3.2. Production of Gas $\left(\mathrm{CO}_{2}\right)$ from Glucose. Upon inoculating the yeast isolates in Yeast Extract Glucose Broth (YEGB) and incubating at $25^{\circ} \mathrm{C}$ for $24 \mathrm{~h}$, from a total of 155 yeast isolates obtained from different fermenting teff dough, only 20 were observed with excellent potential of Gas production from glucose (Table 1).

With the abundance of yeast in relation to sample source most isolates were identified from household and then hotel and followed by microenterprise. Kazachstania bulderi were found from samples collected from household; Saccharomyces cerevisiae and unidentified yeast isolates were found from samples collected from hotel. Candida humilis were found as samples collected from households and
Microenterprise whereas Pichia fermentans were found from all sites.

The yeast isolates were also related to the age of fermentation inoculum used. Some of the yeast were old, some were fresh, and some were both. Candida humilis and Saccharomyces cerevisiae was found from samples having fresh inoculum whereas Kazachstania bulderi were found from samples containing old inoculum. Pichia fermentans and other unidentified yeast isolates were found from samples containing fresh and/or old inoculum.

3.3. Molecular Characterization of the Isolates. The PCR amplification product of 20 yeast isolates of the ITS DNA region has indicated that the yeast isolates recovered in this study have a fragment within 100 to 500 bp (Figure 1) which is important for minimizing the number of isolates manageable for sequencing.

3.3.1. Yeast ITS DNA Sequence Analyses. After all the raw sequences were edited, the BLAST result showed that only 13 samples had significant similarity (98-100\%) to GenBank entries. Comparisons of ITS DNA sequences of the yeast isolates with yeast found in GenBank permitted the identification of the yeast strains. The ITS DNA sequences of the yeast isolates were used for the construction of the phylogenetic tree of yeast isolated from teff dough (Table 2). Sequence similarity was highest (100\%) between AAUYT21B and strain of Pichia fermentans CBS: 2060. Our result indicated that AAUYT25A and AAUYT27A have $100 \%$ similarity with Pichia fermentans strain Pferm 62_08_02_05. AAUYT32A with Pichia fermentans CBS: 4807; AAUYT23C, AAUYT24C, and AAUYT28A with Candida humilis CBS: 8195; AAUYT30B with Saccharomyces cerevisiae strain YAP1; and AAUYT26C and AAUYT34C with Pichia occidentalis strain PMM08-2452L were found to have $99 \%$ similarity. On the other hand, AAUYT31A was shown to have 98\% similarity with Kazachstania bulderi CBS: 8638 .

Accordingly, in this study the yeast isolates were identified as belonging to four genera Candida, Kazachstania, Pichia, and Saccharomyces. The genus Candida consisted of one species, namely, Candida humilis CBS: 8195 (isolates AAUYT23B, AAUYT24C, and AAUYT28). The genus Kazachstania contained one species called Kazachstania bulderi (AAUYT31A), genus Pichia contained two species, namely, Pichia occidentalis (AAUYT26C and AAUYT34C), Pichia fermentans (AAUYT21B, AAUYT25A, AAUYT27A, and AAUYT32A), and genus Saccharomyces contained one species, Saccharomyces cerevisiae (AAUYT30B).

A phylogenetic tree was constructed from amplified and sequenced ITS DNA region of the 13 yeast isolates of those recovered from fermented teff dough in this study, which identified seven species. This phylogenetic grouping indicated that strains having similar sequences were clustered into four genera and nine strains (Figure 2).

The tree was generated through MEGA package version 6 and was used to generate phylogenetic tree based on the Tamura-Nei model for distance estimation and bootstrap analyses with 1,000 replications with a cutoff point of 50 shown next to the branches. The topology of the tree is with 
TABLE 1: Morphological characteristics and gas production of the isolated yeast.

\begin{tabular}{|c|c|c|c|c|c|c|}
\hline \multirow{2}{*}{ Isolates } & \multirow{2}{*}{ Species } & \multicolumn{5}{|c|}{ Colony Morphology } \\
\hline & & Pigmentation & Shape & Elevation & Size & Gas prod 'on \\
\hline AAUYT21B & Pichia fermentans & White & Circular & Flat & Large & ++ \\
\hline AAUYT23B & Candida humilis & White & Circular & Flat & Large & +++ \\
\hline AAUYT24C & Candida humilis & White & Circular & Flat & Medium & +++ \\
\hline AAUYT25A & Pichia fermentans & White & Circular & Flat & Large & + \\
\hline AAUYT26C & Pichia fermentans & Gray & Irregular & Raised & Large & + \\
\hline AAUYT27A & Pichia fermentans & White & Irregular & Raised & Medium & + \\
\hline AAUYT28A & Candida humilis & White & Irregular & Raised & Large & + \\
\hline AAUYT30B & Saccharomyces cerevisiae & Gray & Irregular & Raised & Large & ++ \\
\hline AAUYT31A & Kazachstania bulderi & Blue & Circular & Flat & Large & + \\
\hline AAUYT32A & Pichia fermentans & Blue & Circular & Flat & Large & ++ \\
\hline AAUYT33A & Unidentified & Blue & Circular & Flat & Large & + \\
\hline AAUYT34C & Pichia fermentans & White & Circular & Flat & Large & + \\
\hline AAUYT35B & Unidentified & White & Circular & Flat & Large & + \\
\hline AAUYT36B & Unidentified & Gray & Irregular & Raised & Large & + \\
\hline
\end{tabular}

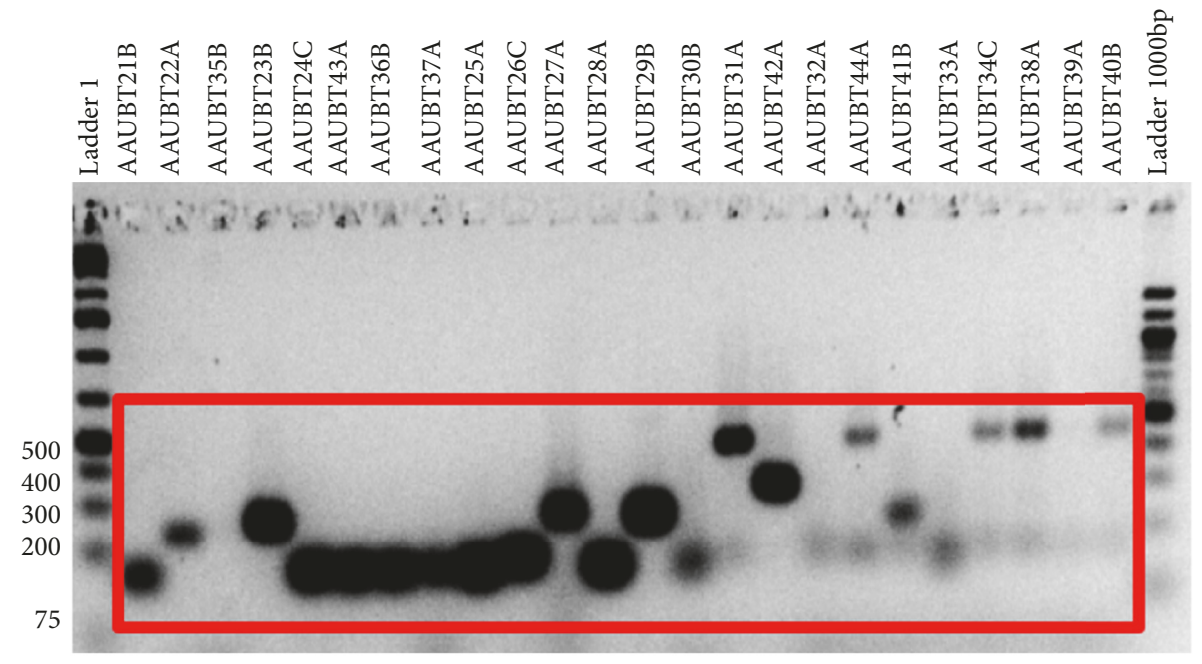

FIGURE 1: PCR amplification of yeast ITS DNA bands amplified by ITS1 and ITS2 yeast.

TABLE 2: Isolates on the basis of similarity to the partial ITS DNA sequence.

\begin{tabular}{lcccc}
\hline Isolates ID & E value & Identity & Species & Accession \\
\hline AAUYT23B & 0.0 & $99 \%$ & Candida humilis CBS:8195 & KY102138.1 \\
AAUYT24C & 0.0 & $99 \%$ & Candida humilis CBS:8195 & KY102138.1 \\
AAUYT28A & 0.0 & $99 \%$ & Candida humilis CBS:8195 & KY103628.1 \\
AAUYT31A & 0.0 & $98 \%$ & Kazachstania bulderi CBS: 8638 & KY104550.1 \\
AAUYT21B & 0.0 & $100 \%$ & Pichia fermentans CBS:2060 & KU987883.1 \\
AAUYT25A & 0.0 & $100 \%$ & Pichia fermentans strain Pferm 62_08_02_05 & KU987883.1 \\
AAUYT27A & 0.0 & $100 \%$ & Pichia fermentans strain Pferm 62_08_02_05 & KY104537.1 \\
AAUYT32A & 0.0 & $99 \%$ & Pichia fermentans CBS:4807 & KP132530.1 \\
AAUYT26C & 0.0 & $99 \%$ & Pichia occidentalis strain PMM08-2452L & KP132530.1 \\
AAUYT34C & 0.0 & $99 \%$ & Saccharomyces cerevisiae strain YAP1 & KY203910.1 \\
AAUYT30B & 0.0 & $99 \%$ &
\end{tabular}




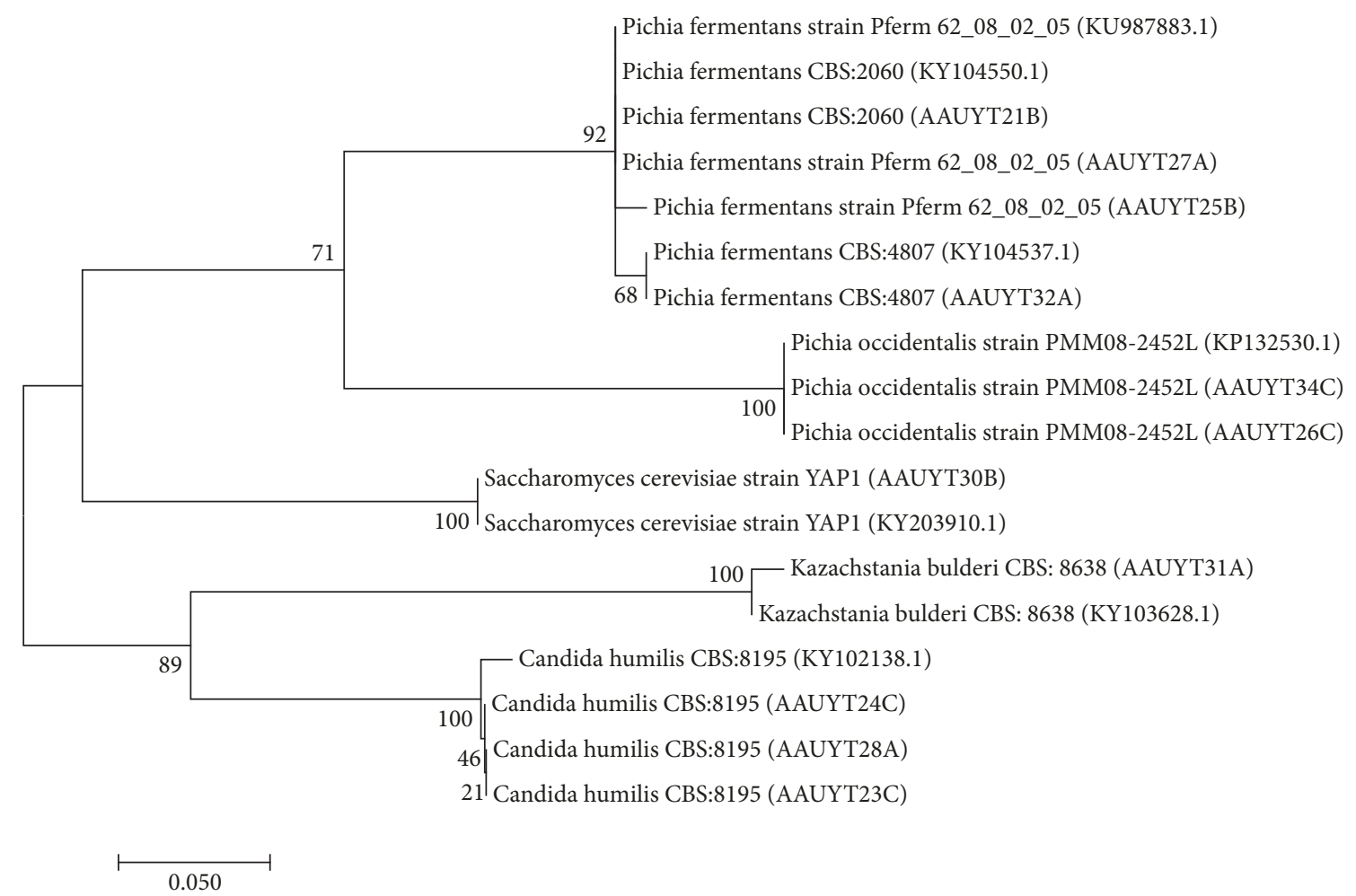

FIGURE 2: Phylogenetic tree of 13 yeast isolates from fermented teff dough using ITS DNA sequence. i.e., 13 yeast isolates were (AAUBT21B, AAUBT25B, AAUBT27A, AAUBT28A, AAUBT24C, AAUBT23C, AAUBT31A, AAUBT26C, AAUBT34C, AAUBT32A and AAUBT30B) and 9 yeast accessions were from GenBank (KY102138.1, KY104888.1, KY104550.1, KU987883.1, KY103628.1, and KY102138.1 AND KY102138.1.).

similarity cutoff point of $50 \%$ and scale length of 0.050 and the highest log likelihood (-430.59). The evolutionary history was inferred by using the Maximum Likelihood method.

\section{Discussion}

This study made a definitive identity of 155 yeast isolates and 20 of these were characterized for their potential in gas production [22]. Yeast isolates with good gas production were believed to be a strong fermenter, preferable for production of Injera, and these were selected for molecular identification.

Molecular identification of yeast from fermented teff dough using sequence data identified was Pichia fermentans, Candida humilis, Kazachstania Saccharomyces cerevisiae, and Pichia occidentalis. Mogessie [17] has indicated that yeast found in dough mainly contained Candida milleri, Rhodotorula mucilaginosa, Kluyveromyces marxianus, and Pichia naganishii. Candida milleri was found from every household. This independent work supports the previous results of Gifawesen and Abraham [14] who indicated that the dominant yeast of fermenting teff dough were species of Torulopsis, Saccharomyces, Candida, and Pichia. Askal and Kebede [10] have indicated that Candida humilis, Saccharomyces cerevisiae, Cryptococcus tropicalis, and Saccharomyces exiguus were found from early stage to the end of teff dough fermentation. In this research also yeast were identified from the beginning of fermentation to the end.

Yeast was identified from all sample sources, i.e., household, hotel, and microenterprises. Kazachstania bulderi were found from household whereas Saccharomyces cerevisiae and unidentified yeast was found in samples collected from hotels. This variation may be due to personal hygiene and the source of the flour used for dough preparation. Unidentified yeast isolates were found from samples collected from both hotels and households. Candida humilis have identified both samples from households and microenterprise whereas Pichia fermentans were found from all sites. Yeast was also identified from samples with fresh and old inoculum and the selected isolates were from each category, i.e., samples containing fresh and/ or old inoculum. Berhanu [15] also concluded that yeast become the predominant group of organisms in ersho [12]. Saccharomyces spp. become abundant and is responsible for the rising of the dough. The yeast most prevalent in the ersho belonged to the genera Candida and Pichia [12, 14]. Saccharomyces spp. and Torulopsis spp. are the dominating group of microorganisms after 50h of fermentation [28]. Those identified yeast may involve in teff dough fermentation during Injera preparation. Knowing the yeast found in teff dough at a species level is important for many aspects of the starter culture in formulation for diverse and selected taste characteristics of teff Injera.

\section{Conclusion}

The study indicated that there is the involvement of different yeast in teff dough fermentation (i.e., Pichia fermentans, Candida humilis, Kazachstania Saccharomyces cerevisiae, and Pichia occidentalis). The identification of these isolates could 
possibly contribute to the information needed for understanding and verification of potential yeast involved in the course of teff dough fermentation.

\author{
Abbreviations \\ bp: Base pair \\ $\mathrm{CO}_{2}$ : Carbon dioxide \\ DNA: Deoxyribonucleic acid \\ ITS: Internal transcribed spacers \\ PCR: Polymerase chain reaction \\ RTA: Ready to prepare \\ RNA: Ribonucleic acid \\ $\mu \mathrm{l}$ : Microliter.
}

\section{Data Availability}

The data used to support the findings of this study are available from the corresponding author upon request.

\section{Conflicts of Interest}

The authors declare that they have no competing interests.

\section{Authors' Contributions}

Mr. Belay Tilahun conducted all the experiments and wrote the manuscript. Mr. Andualem Bahiru, Dr. Anteneh Tesfaye, and Dr. Diriba Muleta coordinated the research work, designed portions of the experiments, and were involved in the initial draft and the revision of the manuscript. Dr. Zewdu Terefework participated in the molecular laboratory work, provided laboratory space, and revised the manuscript. Prof. Gary Wessel performed the DNA cloning and sequencing and revised the manuscript. All authors read and approved the final manuscript.

\section{Acknowledgments}

Mr. Belay thanks Dr. Abiy Zegeye, who have kindly assisted in the sequence analysis and bioinformatics part of the research. Mr. Belay also acknowledges the Holeta Agricultural Biotechnology Research Institute and the MRC-ET Molecular Diagnostics Laboratory, for support to accomplish the molecular experiments. This study was supported by the Institute of Biotechnology, Ministry of Science and Technology, Ethiopia, and MRC-ET Molecular Diagnostics, Addis Ababa, Ethiopia. The cost of sample collection was funded by the Institute of Biotechnology Addis Ababa University. The cost of laboratory consumables and laboratory payments was funded by the Ministry of Science and Technology, Ethiopia. MRC-ET Molecular Diagnostics also supported the cost of some molecular laboratory reagents and DNA extraction kits. GMW and the DNA sequencing were supported by $\mathrm{NIH}$ grant 9R01GM125071.

\section{References}

[1] S. Sieuwerts, F. A. de Bok, J. Hugenholtz, and J. E. van Hylckama Vlieg, "Unraveling microbial interactions in food fermentations: from classical to genomics approaches," Applied and Environmental Microbiology, vol. 74, no. 16, p. 4997, 2008.

[2] W. H. Holzapfel, "Appropriate starter culture technologies for small-scale fermentation in developing countries," International Journal of Food Microbiology, vol. 75, no. 3, pp. 197-212, 2002.

[3] A. Nigatu and B. Abegaz Gashe, "Inhibition of spoilage and food-borne pathogens by lactic acid bacteria isolated from fermenting tef (Eragrostis tef) dough," Ethiopian Medical Journal, vol. 32, no. 4, pp. 223-229, 1994.

[4] A. T. Adesulu and K. O. Awojobi, "Enhancing sustainable development through indigenous fermented food products in Nigeria," African Journal of Microbiology Research, vol. 8, no. 12, pp. 1338-1343, 2014.

[5] O. K. Achi, "The potential for upgrading traditional fermented foods through biotechnology," African Journal of Biotechnology, vol. 4, no. 5, pp. 375-380, 2005.

[6] B. Ogwaro, H. Gibson, M. Whitehead, and D. Hill, "Survival of Escherichia coli O157:H7 in traditional African yoghurt fermentation," International Journal of Food Microbiology, vol. 79, no. 1-2, pp. 105-112, 2002.

[7] L. H. Ledenbach and R. T. Marshall, "Microbiological spoilage of dairy products Compendiumof the microbiological spoilage of foods and beverages," in Food Microbiology and Food Safety, W. H. Sperber and M. P. Doyle, Eds., Springer, New York, NY, USA, 2005.

[8] A. W. Ashiru, O. D. Teniola, N. N. Dibiana, and A. Apena, "Isolation of starch degrading spoilage bacteria from 'Ogi' (fermenting maize starch)," Pakistan Journal of Nutrition, vol. 11, no. 3, pp. 243-246, 2012.

[9] M. S. Ali and Z. Latif, "Molecular characterization of yeast strains isolated from different sources by restriction fragment length polymorphism," Pakistan Journal of Botany, vol. 48, no. 1, pp. 363-370, 2016.

[10] D. Askal and A. Kebede, "Isolation, characterization and identification of lactic acid bacteria and yeast involved in fermentation of Teff (Eragrostis teff)," International Journal, vol. 1, pp. 3644, 2013.

[11] C. P. Kurtzman and J. W. Fell, "Yeast systematics and phylogeny-implications of molecular identification methods for studies in ecology," in Biodiversity and Ecophysiology of Yeasts, The Yeast Handbook, Springer, Berlin, Heidelberg, Germany, 2006.

[12] B. R. Stewart and A. Getachew, "Investigation of the nature of Injera," Economic Botany, vol. 16, pp. 127-130, 1962.

[13] A. I. Angelov, G. Petrova, A. D. Angelov et al., "Molecular identification of yeasts and lactic acid bacteria involved in the production of Beninese fermented food degue," Open Biotechnology Journal, vol. 11, pp. 94-104, 2017.

[14] C. Gifawesen and B. Abraham, "Yeast flora of fermenting teff (Eragrostis teff) dough," SINET: Ethiopian Journal of Science, vol. 5, pp. 21-25, 1982.

[15] A. G. Brhanu, G. Meaza, and B. Abraham, "Teff Fermentation, The role of microorganisms in fermentation and their effect on the nitrogen content of teff,' SINET: Ethiopian Journal of Science, vol. 5, pp. 69-76, 1982.

[16] M. Evelyn, Molecular Characterization and Population Dynamics of Lactic Acid Bacteria during the Fermentation of Sorghum, University of Pretoria, 2009.

[17] M. Ashenafi, "Microbial flora and some chemical properties of ersho, a starter for teff (Eragrostis tef) fermentation," World Journal of Microbiology \& Biotechnology, vol. 10, no. 1, pp. 69-73, 1994. 
[18] K. Bacha, H. Jonsson, and M. Ashenafi, "Microbial dynamics during the fermentation of wakalim, a traditional Ethiopian fermented sausage," Journal of Food Quality, vol. 33, no. 3, pp. 370-390, 2010.

[19] T. Anteneh, M. Tetemke, and A. Mogessie, "Antagonism of lactic acid bacteria against foodborne pathogens during fermentation and storage of borde and shamita, traditional Ethiopian fermented beverages," International Food Research Journal, vol. 18, no. 3, pp. 59-64, 2011.

[20] S. N. Leaw, H. C. Chang, H. F. Sun, R. Barton, J.-P. Bouchara, and T. C. Chang, "Identification of medically important yeast species by sequence analysis of the internal transcribed spacer regions," Journal of Clinical Microbiology, vol. 44, no. 3, pp. 693699, 2006.

[21] L. N. Reinking, K. G. Miller, and J. L. Reinking, "Fermentation, respiration \& enzyme specificity: a simple device \& key experiments with yeast," American Biology Teacher, vol. 56, no. 3, pp. 164-168, 1994.

[22] D. Gevers, G. Huys, and J. Swings, “ Applicability of rep-PCR fingerprinting for identification of Lactobacillus species," FEMS Microbiology Letters, vol. 205, no. 1, pp. 31-36, 2001.

[23] T. J. White, T. Bruns, S. Lee, and J. Taylor, "Amplification and direct sequencing of fungal ribosomal RNA genes for phylogenetics," in PCR Protocols: A Guide to Methods And Applications, M. A. Innis, D. H. Gelfand, J. J. Sninsky, and T. J. White, Eds., pp. 315-322, Academic Press, New York, NY, USA, 1990.

[24] J. D. Thompson, D. G. Higgins, and T. J. Gibson, "CLUSTAL $\mathrm{W}$ : improving the sensitivity of progressive multiple sequence alignment through sequence weighting, position-specific gap penalties and weight matrix choice," Nucleic Acids Research, vol. 22, no. 22, pp. 4673-4680, 1994.

[25] S. F. Altschul, W. Gish, W. Miller, E. W. Myers, and D. J. Lipman, "Basic local alignment search tool," Journal of Molecular Biology, vol. 215, no. 3, pp. 403-410, 1990.

[26] K. Tamura and M. Nei, "Estimation of the number of nucleotide substitutions in the control region of mitochondrial DNA in humans and chimpanzees," Molecular Biology and Evolution, vol. 10, pp. 512-526, 1993.

[27] S. Kumar, G. Stecher, and K. Tamura, "MEGA7: molecular evolutionary genetics analysis version 7.0 for bigger datasets," Molecular Biology and Evolution, vol. 33, no. 7, pp. 1870-1874, 2016.

[28] A. Mogessie, "A review on the microbiology of indigenous fermented foods and beverages of ethiopia," Ethiopian Journal of Biological Sciences, vol. 5, pp. 189-245, 2006. 


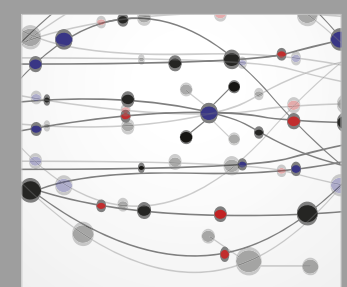

The Scientific World Journal
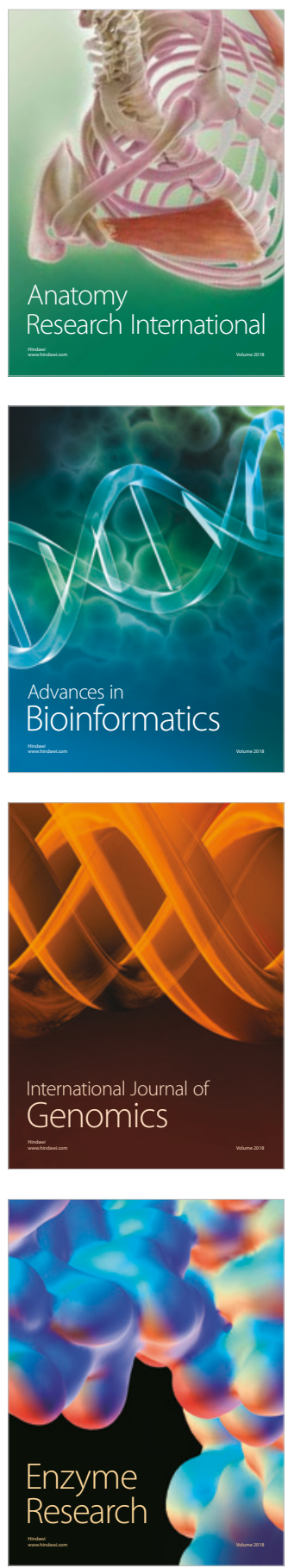
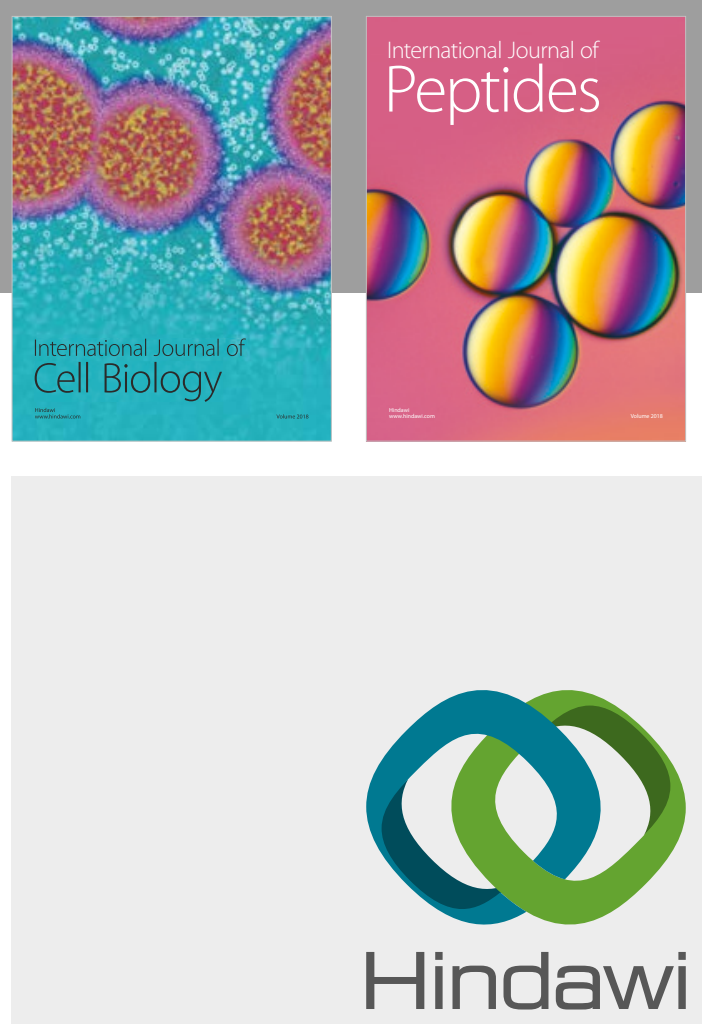

Submit your manuscripts at

www.hindawi.com
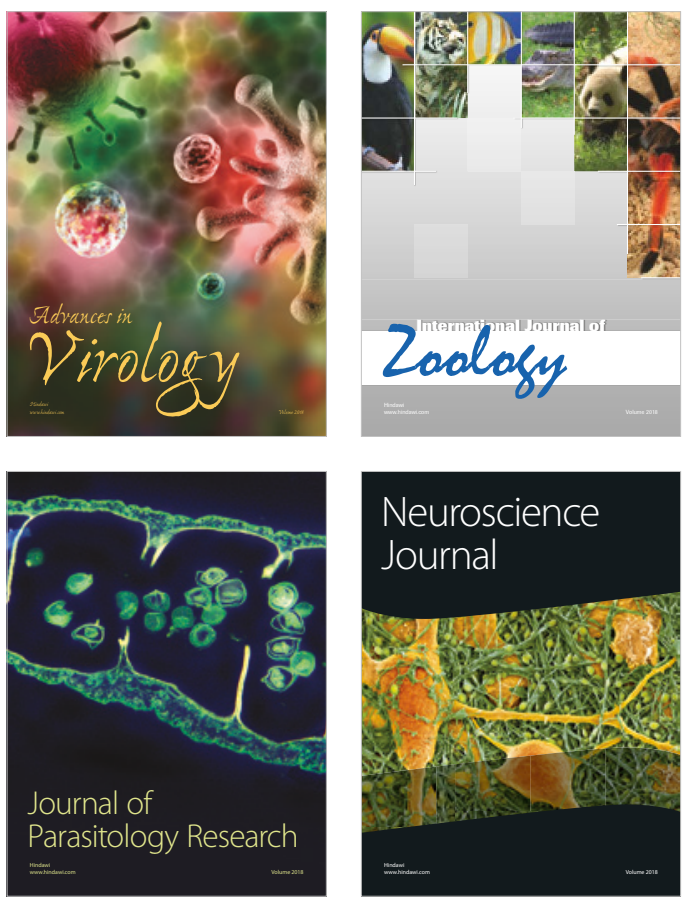
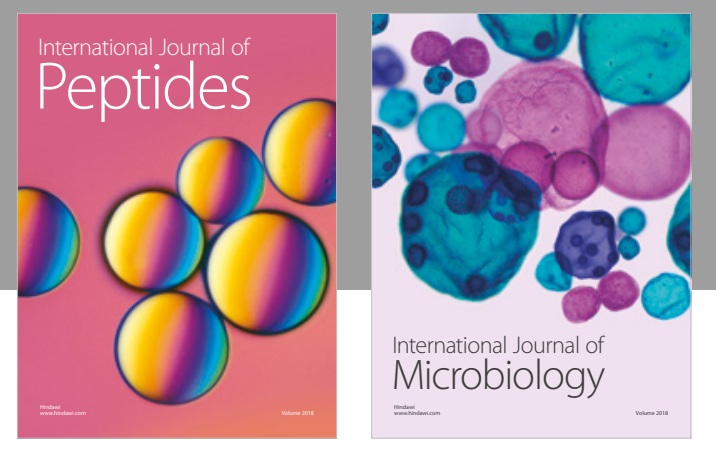

nternational Journal of Microbiology
Journal of
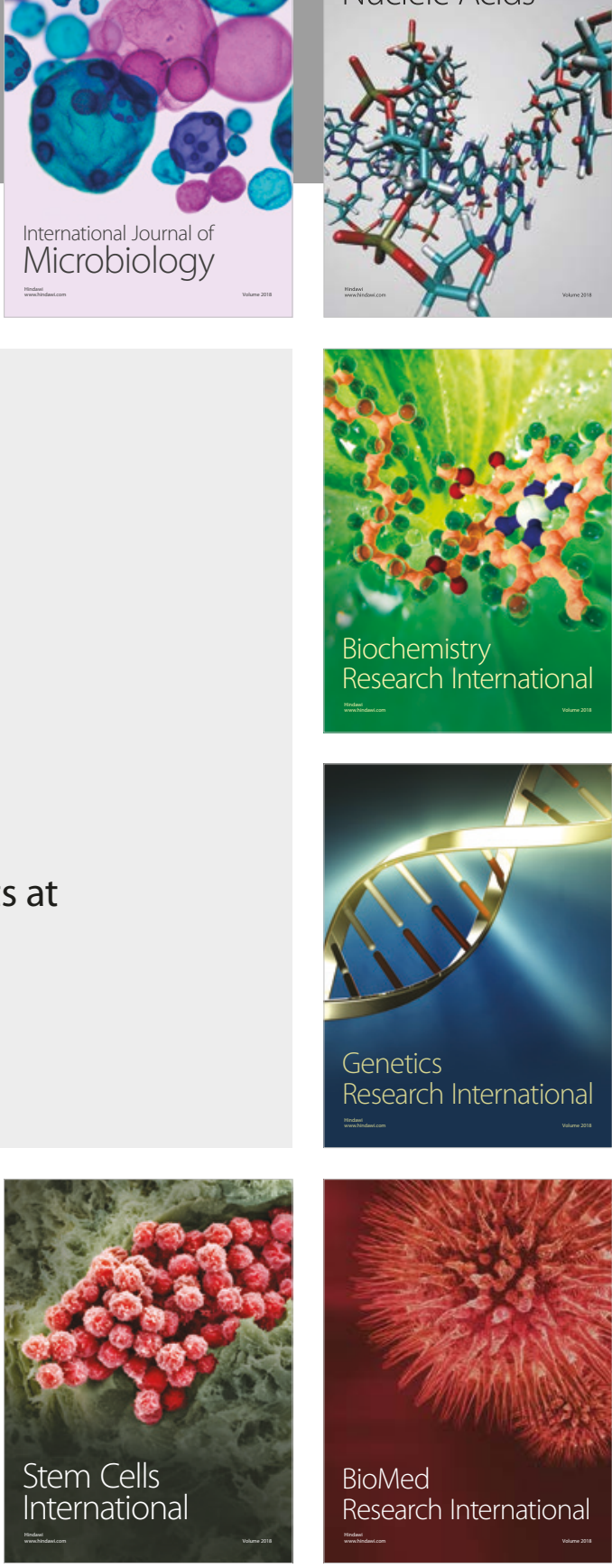
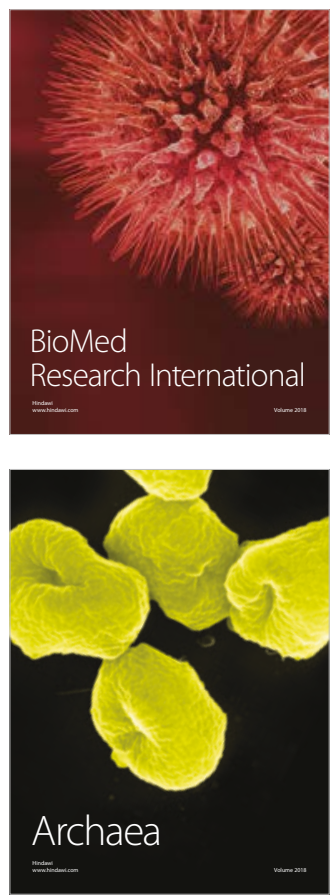